\title{
Video Article \\ Polysome Fractionation and Analysis of Mammalian Translatomes on a Genome-wide Scale
}

\author{
Valentina Gandin ${ }^{1}$, Kristina Sikström ${ }^{2}$, Tommy Alain ${ }^{3}$, Masahiro Morita ${ }^{3}$, Shannon McLaughlan ${ }^{1}$, Ola Larsson ${ }^{2}$, Ivan Topisirovic ${ }^{1}$ \\ ${ }^{1}$ Lady Davis Institute and Department of Oncology, McGill University \\ ${ }^{2}$ Department of Oncology-Pathology, Karolinska Institutet \\ ${ }^{3}$ Goodman Cancer Centre and Department of Biochemistry, McGill University
}

Correspondence to: Ola Larsson at ola.Iarsson@ki.se, Ivan Topisirovic at ivan.topisirovic@mcgill.ca

URL: https://www.jove.com/video/51455

DOI: doi:10.3791/51455

Keywords: Biochemistry, Issue 87, Cells, Eukaryota, Nutritional and Metabolic Diseases, Neoplasms, Metabolic Phenomena, Cell Physiological Phenomena, mRNA translation, ribosomes, protein synthesis, genome-wide analysis, translatome, mTOR, elF4E, 4E-BP1

Date Published: 5/17/2014

Citation: Gandin, V., Sikström, K., Alain, T., Morita, M., McLaughlan, S., Larsson, O., Topisirovic, I. Polysome Fractionation and Analysis of Mammalian Translatomes on a Genome-wide Scale. J. Vis. Exp. (87), e51455, doi:10.3791/51455 (2014).

\section{Abstract}

mRNA translation plays a central role in the regulation of gene expression and represents the most energy consuming process in mammalian cells. Accordingly, dysregulation of mRNA translation is considered to play a major role in a variety of pathological states including cancer. Ribosomes also host chaperones, which facilitate folding of nascent polypeptides, thereby modulating function and stability of newly synthesized polypeptides. In addition, emerging data indicate that ribosomes serve as a platform for a repertoire of signaling molecules, which are implicated in a variety of post-translational modifications of newly synthesized polypeptides as they emerge from the ribosome, and/or components of translational machinery. Herein, a well-established method of ribosome fractionation using sucrose density gradient centrifugation is described. In conjunction with the in-house developed "anota" algorithm this method allows direct determination of differential translation of individual mRNAs on a genome-wide scale. Moreover, this versatile protocol can be used for a variety of biochemical studies aiming to dissect the function of ribosome-associated protein complexes, including those that play a central role in folding and degradation of newly synthesized polypeptides.

\section{Video Link}

The video component of this article can be found at https://www.jove.com/video/51455/

Introduction

The regulatory networks that control gene expression have been extensively studied in the last two decades. The vast majority of these research efforts focused on transcriptional regulation, whereby changes in the steady-state mRNA levels on a genome-wide scale were used to determine so-called "gene expression" profiles. Recent studies reveal that steady-state mRNA levels only loosely correspond to the composition of the proteome $^{1,2}$, thereby indicating that the post-transcriptional mechanisms, including mRNA translation, play a major role in regulation of gene expression $^{3,4}$. Indeed, it has been estimated that $\sim 50 \%$ of protein levels are determined at the level of mRNA translation in immortalized mouse fibroblasts ${ }^{5}$.

mRNA translation is a highly regulated process during which mRNAs are translated into proteins via orchestrated action of ribosomes, transfer RNAs (tRNAs) and accessory factors commonly referred to as translation factors (TFs) ${ }^{6}$. Protein synthesis is the most energy consuming process in mammalian cells ${ }^{7}$ and therefore global mRNA translation rates are adjusted to accommodate nutrient availability and cell proliferation rates $^{8}$. In addition to alterations in global mRNA translation rates, various extracellular stimuli (e.g. hormones and growth factors), intracellular cues (e.g. amino acid levels) and different types of stress (e.g. ER-stress) induce selective changes in the pools of mRNAs that are being translated (translatome) ${ }^{6}$. Depending on the type of stimulus, translation activity of some, but not all mRNAs are dramatically affected, thereby resulting in changes in the proteome that are required to mount a rapid cellular response ${ }^{6}$. These qualitative and quantitative changes in the translatome are thought to be mediated by the interplay between trans-acting factors (e.g. components of translational machinery, auxiliary factors or miRNAs) and cis-elements (RNA elements or features) present in selected subsets of mRNAs,9. For instance, changes in the levels and/or activity of rate-limiting translation initiation factors elF4E and elF2 selectively modulate translation of transcripts based on the specific $5^{\prime}$ UTR features ${ }^{6}$. elF4E and elF2 are required for the recruitment of mRNA and initiator tRNA to the ribosome, respectively ${ }^{6}$. An increase in eIF4E activity selectively bolsters translation of mRNAs harboring long and highly structured 5'UTRs including those encoding proliferation and survival stimulating proteins including cyclins, c-myc and Bcl-xL ${ }^{10}$. In turn, inactivation of elF2 leads to a global protein synthesis shut-down, whilst selectively up regulating translation of mRNAs containing short inhibitory upstream open reading frames (uORFs) in their 5'UTRs, such as those encoding master transcriptional regulators of the unfolded protein response (e.g. ATF4). In response to various stimuli including nutrients, growth factors and hormones, the mechanistic/mammalian target of rapamycin (mTOR) pathway stimulates elF4E activity by inactivating the $4 \mathrm{E}$-binding protein (4E-BP) family of translation suppressors, whereas the MAPK pathway directly phosphorylates elF4E ${ }^{6,11}$. In turn, elF2a kinases (i.e. PERK, PKR, HRI and GCN2) inhibit elF2 by phosphorylating its elF2 $\alpha$ regulatory subunit in response to nutrient deprivation, ERstress and virus infection ${ }^{6,12}$. Alterations in the activity and/or expression of TFs, other components of the translational machinery and regulatory factors including miRNAs have been observed in various pathological states including cancer, metabolic syndromes, neurological and psychiatric 
disorders, and renal and cardiovascular disease ${ }^{13-19}$. Collectively, these data indicate that translational mechanisms play a pivotal role in maintaining cell homeostasis and that their abrogation plays a central role in the etiology of various human diseases.

Herein, a protocol for fractionation of polysomes by sucrose density gradient centrifugation in mammalian cells, which is used to separate polysomes from monosomes, ribosomal subunits and messenger ribonucleoprotein particles (mRNPs) is described. This enables discrimination between efficiently translated (associated with heavy polysomes) from poorly translated (associated with light polysomes) mRNAs. In this assay, ribosomes are immobilized on the mRNA using translation elongation inhibitors such as cycloheximide ${ }^{20}$ and cytosolic extracts are separated on $5-50 \%$ linear sucrose density gradients by ultracentrifugation. Subsequent fractionation of sucrose gradients allows isolation of mRNAs according to the number of ribosomes they bind to. RNA extracted from each fraction can be then used to determine changes in the distributions of mRNAs across the gradient between different conditions, whereby translational efficiency increases from the top to the bottom of the gradient. Northern Blotting or quantitative reverse transcription polymerase chain reaction (qRT-PCR) are used to determine levels of mRNAs in each fraction.

Alternatively, fractions containing heavy polysomes (typically more than 3 ribosomes) are pooled and genome-wide polysome-associated mRNA levels are determined using microarray or deep-sequencing. It is of utmost importance to emphasize that the levels of polysome-associated mRNAs are, in addition to translation, affected by transcriptional and post-transcriptional mechanisms that influence cytosolic mRNAs levels ${ }^{21}$ Therefore, to determine differences in translation using genome-wide data from polysome-associated mRNA it is necessary to correct for the effects from steps in the gene expression pathway that are upstream of translation ${ }^{21}$. To allow such a correction, cytosolic RNA is prepared in parallel with polysome-associated RNA from each sample and the genome-wide steady-state mRNA levels are determined ${ }^{21}$. Currently, so-called "translation-efficiency" (TE) scores (i.e. the log-ratio between polysome-associated mRNA data and cytosolic mRNA data) are often employed to correct for the effects of changes in cytosolic mRNA levels on translation efficiency of a given mRNA ${ }^{22}$. However, using TE scores to identify differential translation is associated with substantial numbers of false positive and false negative findings due to a mathematical property of the TE scores commonly referred to as spurious correlation ${ }^{27}$. Indeed a survey of data sets from multiple labs indicated that such spurious correlations seem to be inevitable when analyzing changes in the translatomes ${ }^{23}$. This prompted development of the "analysis of differential translation" (anota) algorithm, which does not suffer from the aforementioned shortcomings ${ }^{23}$. During anota-analysis a regression model is used to derive measures of translational activity independent of cytosolic RNA levels. Such measures are then compared between conditions and a statistics is calculated. The user has the option of applying a variance shrinkage method, which improves statistical power and reduces the occurrence of false positive findings in studies with few replicates ${ }^{24}$. Significantly, it was recently demonstrated that perturbations in the translatome captured by anota, but not TE scores, correlate with changes in the proteome ${ }^{25}$. Therefore, it is highly recommended to apply anota analysis for identification of changes in translation on a genome-wide scale. While the theoretical underpinnings of the anota algorithm were discussed in detail previously ${ }^{21,23,26}$, here focus is on how to apply it in practice.

In addition to studying changes in mRNA translation activity in the cell, this ribosome fractionation protocol can be used to isolate and biochemically and functionally characterize ribosome- and polysome-associated protein complexes. This approach has successfully been deployed in the past to identify complexes that regulate the stability of newly synthesized polypeptides ${ }^{27}$ and/or are involved in the phosphorylation of components of the translational machinery. This application of the polysome fractionation method will also be briefly discussed.

\section{Sucrose Gradient Preparation}

1. Prepare $100 \mathrm{ml}$ of $60 \%(\mathrm{w} / \mathrm{v})$ sucrose solution in $\mathrm{ddH}_{2} \mathrm{O}$. Solution should be filtered through a $0.22 \mu \mathrm{m}$ filter to prevent clogging of the tubing during the fractionation step (step 3.5).

2. Prepare $5 \mathrm{ml}$ of $10 \mathrm{x}$ sucrose gradient buffer: $200 \mathrm{mM}$ HEPES $(\mathrm{pH} 7.6), 1 \mathrm{M} \mathrm{KCl}, 50 \mathrm{mM} \mathrm{MgCl}, 100 \mu \mathrm{g} / \mathrm{ml}$ cycloheximide, $1 \times$ protease inhibitor cocktail (EDTA-free), 100 units/ml RNase inhibitor.

3. Using the $60 \%$ solution prepared as described in step 1.1 , make $40 \mathrm{ml}$ of $5 \%$ and $50 \%$ sucrose solutions in $1 \times$ sucrose gradient buffer.

4. Use the Marker block provided with the gradient maker to mark the half-full point on each polyallomer ultracentrifugation tube for layering (6 ultracentrifugation tubes in total). Using the layering device provided with the gradient maker, add $5 \%$ sucrose solution until it reaches the half-full point. Then, add $50 \%$ sucrose solution from the bottom until the interface between the two solutions reaches the half-full point. Special care should be taken during this step so that gradients are as similar as possible, as this is essential for obtaining high reproducibility (see below).

5. Seal tubes with rate zonal caps provided with the gradient maker and transfer the sealed tubes to the tube holder on the gradient maker.

6. Run the gradient maker to obtain a linear $5 \%$ to $50 \%$ gradient. 6 linear gradients will be formed in few minutes by rotation at high tilt angle. Note: Sucrose gradients can be used immediately or stored at $-80^{\circ} \mathrm{C}$ for 6 months.

\section{Isolation and Sedimentation of Polysomes}

1. Depending on the cell type, seed cells into $1-515-\mathrm{cm}$ Petri dishes $\left(\sim 15 \times 10^{6}\right.$ cells $)$ at least one day before lysis. On the day of the experiments cells should be $\sim 80 \%$ confluent. Note: Optimal confluency is critical because translation and proliferation rates are directly proportional $^{8}$, and therefore polysomes should be analyzed in actively proliferating cells (i.e. translation rates will significantly drop in over confluent cells whereas low confluency of cells may not provide sufficient material for further analysis). Exceptions of this rule can be made if for instance the experimenter is interested in studying the effect of contact inhibition on translation. However, cells should not be kept under over confluent conditions for too long, as this may have inadvertent effects on the translatome.

If transfection is required, most of the commercially available kits do not affect polysome levels. Because on the day of transfection cells must be $80-90 \%$ confluent, it is recommended to propagate cells $24 \mathrm{hr}$ post-transfection and isolate polysomes $48 \mathrm{hr}$ post-transfection from cells $\sim 80 \%$ confluent. 
2. Incubate cells with cycloheximide at a final concentration of $100 \mu \mathrm{g} / \mathrm{ml}$ in growth media for $5 \mathrm{~min}$ at $37{ }^{\circ} \mathrm{C}$ and $5 \% \mathrm{CO}_{2}$; and wash cells twice with $10 \mathrm{ml}$ of ice-cold $1 \times$ PBS containing $100 \mu \mathrm{g} / \mathrm{ml}$ cycloheximide. Cycloheximide is a translation elongation inhibitor that "freezes" ribosomes on the mRNA, thereby preventing ribosome run off ${ }^{20}$.

3. Scrape cells gently, in $5 \mathrm{ml}$ of ice-cold $1 \times$ PBS containing $100 \mu \mathrm{g} / \mathrm{ml}$ cycloheximide and collect them in a $50 \mathrm{ml}$ tube. It is important that this step is performed reasonably fast as leaving cells on ice for a prolonged period of time will drastically decrease the quality of the polysome preparation.

4. Collect cells by centrifugation at $200 \times \mathrm{g}$ for $5 \mathrm{~min}$ at $4{ }^{\circ} \mathrm{C}$.

5. Discard supernatant and resuspend cells in $425 \mu \mathrm{l}$ of hypotonic buffer $[(5 \mathrm{mM}$ Tris- $\mathrm{HCl}(\mathrm{pH} 7.5), 2.5 \mathrm{mM} \mathrm{MgCl} 2,1.5 \mathrm{mM} \mathrm{KCl}$ and $1 \times$ protease

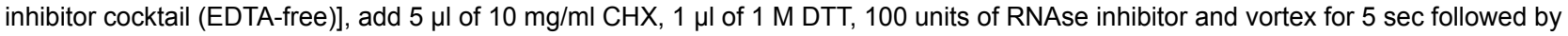
addition of $25 \mu \mathrm{l}$ of $10 \%$ Triton X-100 (final concentration $0.5 \%$ ) and $25 \mu \mathrm{l}$ of $10 \%$ Sodium Deoxycholate (final concentration $0.5 \%$ ) and vortex for $5 \mathrm{sec}$. Due to cell swelling, the hypotonic buffer will disrupt the plasma membrane, whereas the mild detergent conditions are used to solubilize cytosolic and endoplasmic reticulum-associated ribosomes without disrupting the nuclear envelope.

6. Centrifuge lysates at $16,000 \times \mathrm{g}$ for $7 \mathrm{~min}$ at $4{ }^{\circ} \mathrm{C}$ and transfer supernatant $(\sim 500 \mu \mathrm{l})$ to a new pre-chilled $1.5 \mathrm{ml}$ tube. Measure OD at $260 \mathrm{~nm}$ for each sample using a spectrophotometer and keep $10 \%$ of the lysate as input that will be used to determine cytosolic steady-state mRNA levels. Dilute the input to $750 \mu \mathrm{l}$ in RNAse free $\mathrm{H}_{2} \mathrm{O}$, add $750 \mu \mathrm{l}$ of Trizol and flash freeze in liquid nitrogen.

7. Transfer ultracentrifuge tubes containing sucrose gradients in pre-chilled rotor buckets. Remove $500 \mu$ from the top of sucrose gradients. Adjust lysates so that they contain the same OD (10-20 OD at $260 \mathrm{~nm}$ ) in $500 \mu \mathrm{l}$ of lysis buffer (described in step 2.5) and load them onto each sucrose gradient.

Note: It is important to immediately load lysates on the gradients, as this will critically improve the quality of polysome preparations.

8. Weigh and balance each gradient before the ultra-centrifugation.

9. Centrifuge at $222,228 \times \mathrm{g}(36,000 \mathrm{rpm})$, for $2 \mathrm{hr}$ at $4{ }^{\circ} \mathrm{C}$ using SW41Ti rotor. Note: In order not to disrupt sucrose gradients, "low" brake option should be selected.

\section{Polysome Fractionation and RNA Extraction}

1. Prepare the fraction collector by cleaning it with warm RNAse free water containing RNase ZAP (a few sprays). Repeat this step with warm RNAse free water only. Switch UV lamp and wait for the green light to appear.

2. Carefully remove tubes from the rotor and place them on ice. Switch on computer, pump, UV detector and fraction collector. Set pump at 3 $\mathrm{ml} / \mathrm{min}$ and fill the tubing with the chasing solution $[60 \%(\mathrm{w} / \mathrm{v})$ sucrose containing $0.02 \%(\mathrm{w} / \mathrm{v})$ bromophenol blue] until it reaches the needle. Make sure to see at least one drop coming out of the needle, and ascertain that no bubbles are introduced in the pump syringe or tubing

3. Place $2 \mathrm{ml}$ tubes in a fraction collector. Launch analysis program and set sensitivity to $+/-10 \mathrm{mEV}$. Set "timebase" to $100 \mathrm{sec}$.

4. Position each ultracentrifugation tube into the UV detector while making sure that the tube is in the orthogonal position. Pierce the tube with the needle by twisting the knob below the tube holder.

5. Set the pump at $1.5 \mathrm{ml} / \mathrm{min}$ and collect the fractions setting the time to $30 \mathrm{sec}$ on the fraction collector (this will result in $\sim 750 \mu \mathrm{l}$ in each fraction). Put the pump in the "remote position", start the pump and fraction collector, and at the same time start recording using the DAQ tracer. This will start an upward displacement of the sucrose gradient and a simultaneous detection of UV absorbance at $254 \mathrm{~nm}$. Stop collecting as soon as the first drop of chasing solution falls in a $2 \mathrm{ml}$ collecting tube.

6. Save the tracing in .csv format and (once step 3.7 is completed), in a spreadsheet application, do the following to identify the positioning of each fraction in the UV absorbance profile:

1. Select the column containing values corresponding to absorbance at $254 \mathrm{~nm}$ (channel 0 ).

2. Create a smooth line scatter plot of the absorbances.

3. Set the major unit of the $X$ axis to 300 (value obtained by dividing the volume of the $5 \%-50 \%$ sucrose gradient ( $12 \mathrm{ml}$ ) by the collection time of each fraction $(30 \mathrm{sec})$.

7. Add $750 \mu \mathrm{l}$ of Trizol in each fraction and flash freeze the fractions in liquid nitrogen.

8. Isolate polysome-associated and cytosolic (from 2.6) RNA using Trizol protocol according to manufacturer's instructions. To increase RNA yield, proceed with RNA precipitation at $-80^{\circ} \mathrm{C}$ for 30 min or $-20^{\circ} \mathrm{C}$ overnight.

Note: Because the amount of RNA precipitated from polysomal fractions may not be clearly visible it is recommended to add carrier. Add $1 \mu \mathrm{l}$ of carrier to the tube before the RNA precipitation step during the Trizol protocol.

9. Determine which fractions correspond to mRNA associated with >3 ribosome (using approach described 3.6) and pool these fractions. Use the RNA cleanup kit to perform cleanup of pooled polysome-associated and cytosolic RNA (from 2.6). Submit samples to a facility to determine genome-wide mRNA levels using microarrays or deep-sequencing.

If translation of specific mRNAs need to be monitored by RT-qPCR, it is recommended to use 500 ng of RNA (from pooled fractions containing $>3$ ribosomes or total RNA) to synthesize cDNA.

Note: mRNAs associated with $>3$ ribosomes is set arbitrarily to represent efficiently translated mRNAs and contain more than $80 \%$ of newly synthesized polypeptides ${ }^{28,29}$. Including fractions corresponding to light (1-2), medium (2-3) and heavy polysomes (>3) would be advantageous, but these will increase number of the samples by 2 -fold ( 4 vs 2 per condition) and thus the cost of the experiment. Notwithstanding that it is anticipated that the decrease in cost of deep-sequencing and microarray analysis in future, should favor the latter approach, it is advised that during validation, distribution of individual mRNAs is monitored in each fraction.

\section{Genome-wide Analysis of mRNA Translation}

1. Install $\mathrm{R}$, bioconductor and the anota package:

1. Install R (download from r-project.org) and start an R-session.

Note: $R$ is available for all operating systems and anota will run on all of them.

2. Install bioconductor (bioconductor.org). $\mathrm{R}$ code is interpreted through the R-terminal (e.g. the R console in Windows - which is launched by double clicking the R shortcut). Bioconductor is installed by typing (in the R-terminal): source("http://bioconductor.org/biocLite.R") biocLite() 
3. Install the anota bioconductor package (and the qvalue package that anota requires) by typing (in the R-terminal): source("http://bioconductor.org/biocLite.R") biocLite(c("anota", "qvalue"))

4. Test that the package was successfully installed and open the anota manual by typing (in the R-terminal): library("anota") vignette("anota")

5. Note: Additional help for all functions that are used in the anota package can be found either at the anota web-page (http:// www.bioconductor.org/packages/release/bioc/html/anota.html) or by using the help function within R. For example, to get help on the anotaPerformQc function go to the R-terminal and type (note that this only works when the anota package has been loaded): library("anota") \#loads the anota package ?anotaPerformQc \#opens help for this function

2. Import data to R:

1. Prepare expression data for analysis. Data should be pre-processed (e.g. normalized and quality controlled) with respect to the technique that was used to measure the mRNA levels. Input values must be log transformed, most common is log2. Compile one table with data for all polysome-associated RNA samples and one for all cytosolic RNA samples. The first column should contain the gene-identifiers followed by one data column per sample; the first row should include names for the samples. It is critical that the tables have identical sample order and identical gene order. Save the files as tab-delimited text files called "myPolysomeData.txt" and "myCytosolicData.txt".

2. In this example two sample classes are used (control or diseased) with 3 replicates per class, thereby generating 6 samples with this order: C1, C2, C3, D1, D2, D3 in both the myCytosolicData.txt and myPolysomeData.txt files. Anota requires at least 3 replicates per condition when there are two sample classes. These should be independent biological replicates.

3. Create a directory that contains the data input files (myPolysomeData.txt and myCytosolicData.txt). Open R from this directory. In Windows/Mac this can be done by copying an R-shortcut into this directory and launching $\mathrm{R}$ using this shortcut (the working directly can also be changed using the drop-down menus).

4. Create a file called myCode.R within the newly created directory and open it using a text editing software. Write the code in this file.

5. To load the data into $R$ write the following code to the myCode. $R$ file (remember to re-save this file after each addition of code). Below is a step-by-step explanation of the code but all code could also be written initially and the source function (that runs the code, see below) used only once:

\#\#this loads the anota library

library(anota)

\#\#This loads input data into $R$

dataCyto <- as.matrix(read.table("myCytosolicData.txt", header=TRUE, row.names=1, sep="|t"))

dataPoly <- as.matrix(read.table(“myPolysomeData.txt", header=TRUE, row.names=1, sep="|t"))

6. Run the code in $\mathrm{R}$ by typing directly into the $\mathrm{R}$ terminal: source(“myCode.R”)

7. Check that the data was loaded successfully by typing (into the R-terminal):

head(dataCyto) \#will show top of the dataCyto table

head(dataPoly)

3. Identification of differentially translated genes:

1. Generate a vector that describes the sample categories (a vector is a type of object in R). This vector should reflect the sample order in the myPolysomeData.txt and myCytosolicData.txt so that all three objects have an identical order of the samples. The vector will be used when instructing anota about which samples to compare. Add the following to the myCode.R file:

\#\#Note that the replicate samples have identical sample class

\#\#descriptions (i.e. "C" or "D")in this vector.

myPhenotypes <- c("C", "C", "C", "D", “D”, "D")

2. Perform quality control of the data set. There are a number of quality measures that need to be assessed before anota can be used for analysis. These are discussed in detail in the anota manual. Add this code to myCode. $R$ file and save: anotaQcOut <- anotaPerformQc(dataT=dataCyto, dataP=dataPoly, phenoVec=myPhenotypes) anotaResidOut <- anotaResidOutlierTest(anotaQcObj=anotaQcOut)

3. Run the code by typing into R-terminal: source("myCode.R")

This will generate a number of output files, which can be examined as instructed in the anota manual.

4. Identify differentially translated genes. Samples will be compared based on the alphabetical order of the names supplied to the "phenoVec" parameter (i.e. the "myPhenotypes" vector) unless they are specified by the user (using the "contrasts" parameter). Add the following code to myCode.R file and finish by using the "source" command inside the R-terminal as described above: anotaSigOut <- anotaGetSigGenes(dataT=dataCyto, dataP=dataPoly, phenoVec=myPhenotypes, anotaQcObj=anotaQcOut)

5. Use the help for anotaGetSigGenes to understand how the output is formatted and see how to choose sample categories to compare by typing (in the R-terminal):

?anotaGetSigGenes 
6. Filter and plot genes that are differentially translated. There are multiple thresholds that can be applied within the anotaPlotSigGenes function and using help will simplify a custom combination of such thresholds. To select for reliably analyzed genes apply the minSlope $(-0.5)$, maxSlope (1.5) and slopeP (0.01) settings.

Note: In addition, settings applying to RVM ${ }^{23,26,30}$ false discovery rate (FDR) (0.15) and fold changes (log2[1.5]) may e.g. be used to identify differentially translated genes. Other filters such as "selDeltaPT" can be useful for a stricter filtering (e.g. setting selDeltaPT to $\log 2[1.5])$. It is also necessary to specify which comparison should be filtered (by using the selCont argument). Apply the described settings by adding the following line to the myCode.R file:

anotaSigFiltered <- anotaPlotSigGenes(anotaSigObj=anotaSigOut, selContr=1, minSlope=(-0.5), maxSlope=1.5, slopeP=0.01, maxRvmPAdj=0.15, minEff=log2(1.5), selDeltaPT $=\log 2(1.5))$

7. Perform the analysis by using the source function from the R-terminal as described above. This will also generate a graphical output. Examining this output is a good starting point to assess the performance of the analysis and identify any necessary changes to the settings.

8. Generate an output table. Add the following code to the myCode.R file: write.table(anotaSigFiltered\$selectedRvmData, file="MySignificantGenes.txt", sep="|t")

9. Run the code using the source function in the R-terminal. The columns in the resulting table are explained in the help for the anotaPlotSigGenes function.

\section{Representative Results}

mTOR is a major node of the cellular network that coordinates global protein synthesis rates with nutrient availability ${ }^{19}$. mRNA translation is regulated mainly at the rate-limiting initiation step ${ }^{6}$. The proportion of ribosomes engaged in polysomes positively correlates with translation initiation rates ${ }^{28}$. An example of applying the polysome fractionation method to investigate the role of mTOR signaling in mediating the effects of insulin on mRNA translation is presented. To this end, MCF7 human breast cancer cells were maintained in low serum and then stimulated with insulin alone or in combination with the active-site mTOR inhibitor Torin1. Non-stimulated cells that were continuously kept in low serum, served as a control. mRNPs, monosome (80S) and polysome fractions were separated using the polysome fractionation method. Relative to the control cells, insulin induced an increase in absorbance in gradient fractions corresponding to polysomes, accompanied by a concomitant decrease in absorbance in the monosome fraction (Figure 1). These findings show that the proportion of ribosomes engaged in polysomes is increased in insulin treated as compared to control cells, therefore indicating that, as expected, insulin stimulates global translation initiation rates. Torin1 reversed the effects of insulin on absorbance profiles (Figure 1), thereby corroborating the findings that mTOR signaling plays a major role in mediating the effects of insulin on the translation machinery ${ }^{19}$.

Based on a tenet that the inconsistencies in gradient preparation cannot be avoided, questions have been raised regarding the reproducibility of the data obtained using the polysome fractionation method ${ }^{22}$. To empirically test this potentially deleterious issue, cytosolic and heavy polysome-associated RNA (mRNA associated with 4 and more ribosomes) were isolated from MCF7 cells treated with insulin alone or insulin in combination with Torin 1 from 4 independent biological replicates (Figure 2). The effects of insulin and Torin1 on the composition of cytosolic and heavy polysome-associated mRNA in each replicate was determined on a genome-wide scale using a microarray approach. To determine the reproducibility of the polysome fractionation method the principal component analysis (PCA) was applied. Such analysis showed that samples belonging to each condition were tightly positioned within the two first components while the different conditions were well separated (Figure 2). These findings show that the polysome fractionation method as described here is highly reproducible and therefore appropriate to study quantitative and qualitative changes in translation on a genome-wide level. 


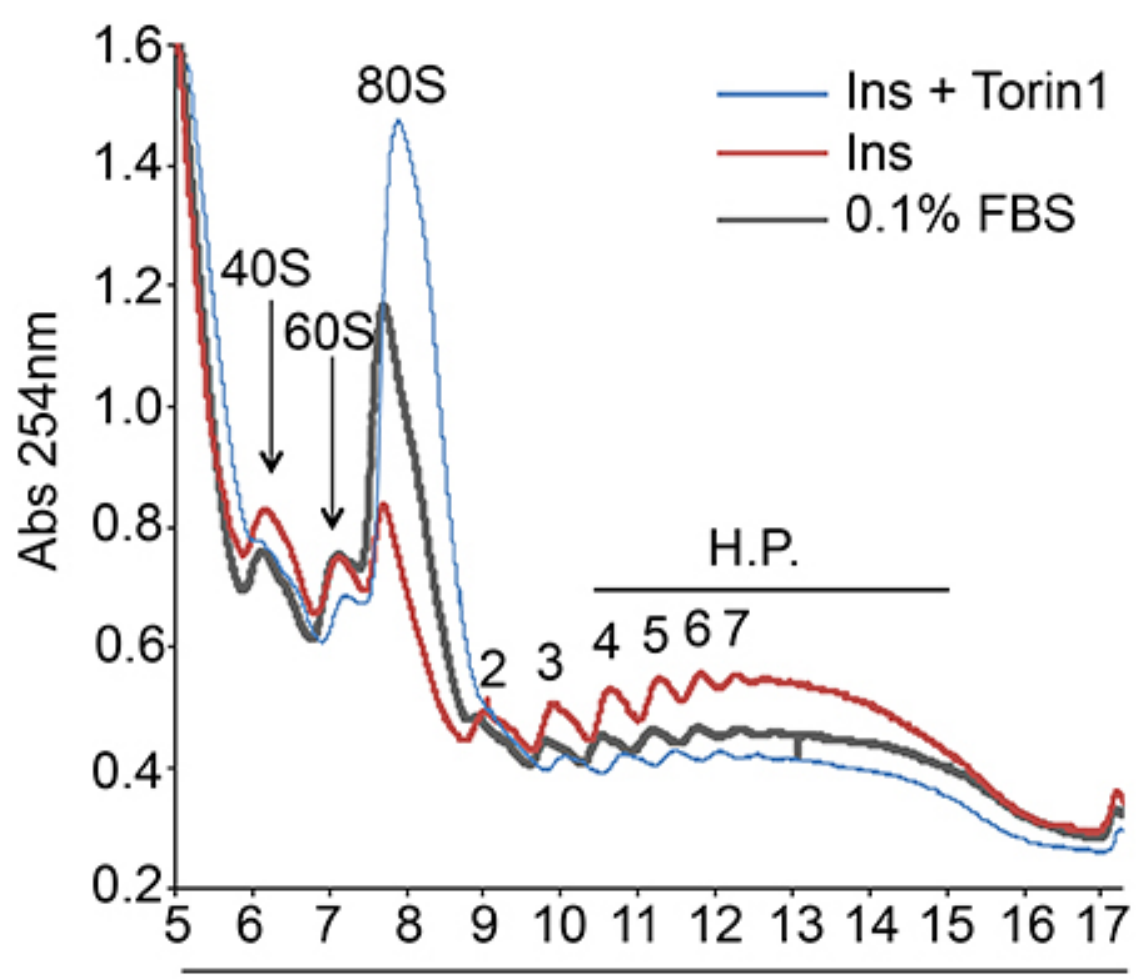

Fraction numbers

$5 \%$

$50 \%$

Figure 1. Polysomal profiles showing the effects of serum starvation, insulin and mTOR signaling on global translation in MCF7 cells. MCF7 cells were deprived of nutrients (maintained in $0.1 \%$ FBS) for 16 hours and treated with $5 \mathrm{nM}$ insulin (Ins) alone or in combination with $250 \mathrm{nM}$ Torin1 (Ins + Torin1) for 4 hours. Untreated cells that were continuously deprived of nutrients $(0.1 \%$ FBS) were used as a control. The corresponding cytosolic extracts were sedimented by centrifugation on $5-50 \%$ sucrose gradients. Free ribosomal subunits (40S and $60 \mathrm{~S}$ ), monosomes (80S) and number of ribosomes in the polysome fractions are indicated. 


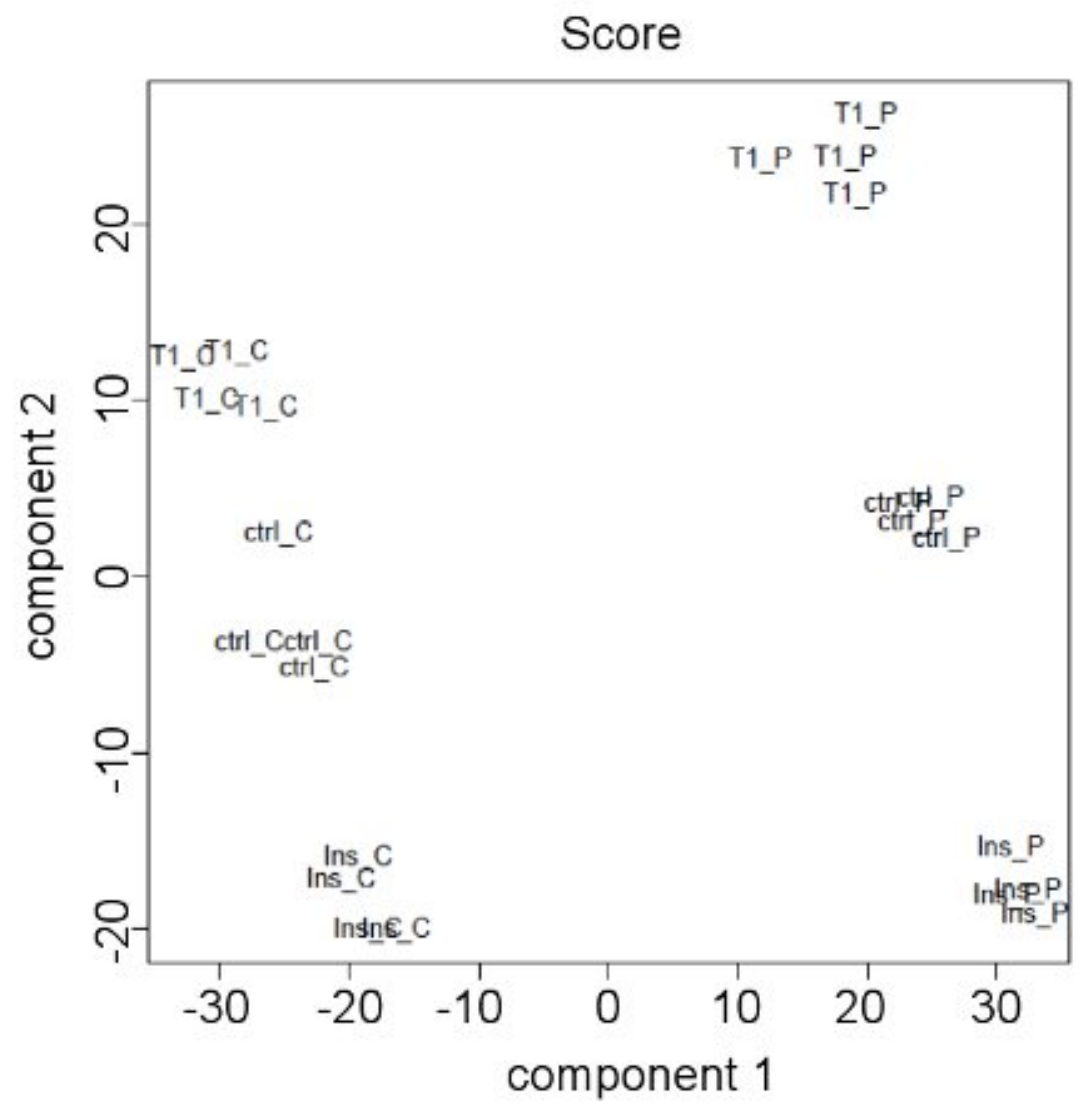

Figure 2. Genome-wide data obtained using polysome profiling is highly reproducible. MCF7 cells were treated as in Figure 1. Cytosolic and polysome-associated RNA (>3 ribosomes) was extracted from 4 independent biological replicates and their genome-wide mRNA levels were determined using GeneTitan arrays (Affymetrix). PCA was used to assess the reproducibility of the resulting data. Shown are the first two PCA components for all treatments ( $\mathrm{T} 1=$ Torin 1 ; ctrl =control; Ins = insulin), origin of RNA ( $=$ cytosolic; $P=$ polysome-associated) and replicates. Samples from the same condition and RNA origin are closely positioned indicating high reproducibility.

\section{Discussion}

This article describes a well-established polysome fractionation protocol followed by an in-house developed analysis method for capturing qualitative and quantitative changes in translational activity on a genome-wide scale in mammalian cells. For successful completion of this protocol special attention should be paid to 1) Cell confluency (as the proliferation rates and nutrient availability correlate with mRNA translation activity and can affect the composition of the translatome, cell confluency should be consistent across replicates and experimental conditions), 2) Rapid cell lysis (Notwithstanding the presence of cycloheximide and RNAse inhibitors in buffers, lysis should be swift and cellular extracts should be overlaid on sucrose gradients immediately after lysis to prevent RNA degradation and dissociation of polysomes), 3) Gradient preparation (to ensure high data reproducibility, sucrose gradients should be prepared using the gradient maker and special care should be taken when handling them).

In addition to studying the changes in translational activity, this protocol can be used to isolate ribosome-associated protein complexes and establish their physiological functions. To this end, levels and phosphorylation status of various ribosome-associated proteins can be determined by TCA precipitation, followed by Western blotting, whereas ribosome-associated protein complexes can be immunoprecipitated from gradient fractions and analyzed by Western blotting or mass-spectrometry. A shortcoming of this technique is that the slow gradient fraction method could result in dissociation of even tightly bound proteins whose binding kinetics are in rapid association/dissociation. Factors associated to newly synthesized polypeptides are typical examples. Newly synthesized polypeptides emerging form the ribosomes can be immobilized on protein complexes associated with ribosomes using chemical cross-linkers such as $3,3^{\prime}$-dithiobis[sulfosuccinimidylpropionate] (DTSSP) ${ }^{31}$. This approach revealed that the Receptor for Activated C Kinase 1 (RACK1)/c-Jun N-terminal kinase (JNK)/eukaryotic translation elongation factor $1 \mathrm{~A} 2$ (eEF1A2) complex regulates degradation of newly synthesized polypeptides in response to stress ${ }^{27}$. In addition, similar methodology was used in studies showing that the protein kinase $\mathrm{C}$ bll (PKCbII) is recruited to ribosomes by RACK $1^{32}$ and that activation of mTOR complex 2 (mTORC2) occurs on the ribosomes where it phosphorylates newly synthesized AKT polypeptides and regulates their stability 33,34 .

Major limitations of the polysome fractionation method followed by anota analysis are: 1$)$ requirement for a relatively high number of cells $(\sim 15$ $x 10^{6}$ cells), 2) lack of positional information regarding localization of ribosome on a given mRNA molecule, and 3 ) issues related to cellular and molecular heterogeneity of normal and tumor tissues. Issues related to required cell number can be resolved by aligning absorbance spectra peaks corresponding to monosomes (80S) of cells whose amounts are limiting with those obtained from high-abundance cells (e.g. HeLa cells) 35 . Ribosome profiling technique (see below) can be used to determine exact position of the ribosome on a given mRNA molecule, whereas 
issues related to confounding effects of tissue heterogeneity on the interpretation of changes in gene expression obtained from complex systems such as human tissues are discussed in detail in a publication by Leek and Storey ${ }^{36}$.

Recently, a novel ribosome profiling technique was developed, wherein ribosome protected fragments (RPFs) are generated by RNAse I treatment and analyzed by deep-sequencing ${ }^{22}$. This technique allows determination of the ribosome position at a single nucleotide resolution, thereby providing hitherto unprecedented insights into ribosome biology. For example, ribosome profiling can be used for determination of ribosome density on a given mRNA molecule or the identification of elements that influence translation initiation rates such as the alternative initiation sites, initiation at non-AUG codons and regulatory elements such as uORFs. However, there are several methodological limitations that restrict the ability of ribosome profiling to accurately estimate mRNA translation efficiency. These include independent biases introduced by random fragmentation and RNAse I digestion, biases introduced by translation inhibitors (e.g. elongation inhibitors such as emetine and cycloheximide are likely to induce ribosome accumulation at translation initiation sites), a large number of false positive and false negative results associated with TE scores as well as their inaccuracy in predicting the reads that originate from protein coding mRNAs ${ }^{37}$. Perhaps most importantly, whereas ribosome profiling allows direct identification of ribosome position on a given mRNA molecule, the number of ribosomes that is associated with a given mRNA is indirectly estimated by normalizing frequencies of reads in RPFs (ribosome-associated mRNA) over those observed in randomly fragmented mRNAs (total mRNA). For instance, in a simple setting where four "B" mRNA molecules (Ba, Bb, Bc and $\mathrm{Bd}$ ) are occupied by four ribosomes at the positions 1, 2, 3 and 4, an inherent shortcoming of the ribosome profiling technique will not permit a distinction between a scenario where all 4 ribosomes associate only with a Ba mRNA in positions 1, 2, 3, and 4 and a scenario where Ba, Bb, $\mathrm{Bc}$ and Bd mRNA are each occupied by a single ribosome at the position 1, 2, 3, and 4, respectively. In contrast, during polysome fractionation, polysome integrity is preserved, thereby allowing isolation of pools of mRNAs associated with a defined number of ribosomes (Figure 1). This important distinction between polysome fractionation and ribosome profiling suggests that whereas the former method can be used to directly compare mRNAs in mRNP, light and heavy polysome fractions, the latter method will likely fail to capture changes in the translatome that are caused by mRNAs that transition from light to heavy polysomes, while overestimating the contribution of those that shift from the mRNP fraction to heavy polysomes. The biological significance of these discrepancies between the aforementioned methods is underscored by a large body of data showing that translational activation of a subset of mRNAs such as those that are elF4E-sensitive, transition from light to heavy polysomes, whereas others, such as those harboring 5 'TOP elements, are recruited to heavy polysomes directly from pools of ribosome-free mRNA 6 . Interestingly, the mTOR pathway has been shown to concomitantly modulate translation of "elF4E-sensitive" and 5'TOP mRNAs ${ }^{11}$. Therefore, methodological differences that are discussed above may explain the apparent discordance of the conclusion between studies using ribosome profiling $^{38,39}$ and polysome fractionation ${ }^{24}$ to assess the effects of mTOR inhibition on the translatome.

In conclusion, polysome fractionation and ribosome profiling are complementary methods that primarily provide information regarding the number of ribosomes associated with mRNA and position of the ribosome on mRNA, respectively. Importantly, notwithstanding the shortcomings and advantages of these methods, it remains essential that the genome-wide data obtained by both procedures are adequately analyzed and functionally and biochemically validated.

\section{Disclosures}

The authors declare no competing financial interests.

\section{Acknowledgements}

This research was supported by the Canadian Institutes of Health Research grant (CIHR MOP-115195) and FRQ-S to I.T., who is also a recipient of CIHR Young Investigator Award; and the Swedish Research Council and the Swedish Cancer Society to O.L.

\section{References}

1. Maier, T., Guell, M., \& Serrano, L. Correlation of mRNA and protein in complex biological samples. FEBS Lett. 583, 3966-3973, doi:S0014-5793(09)00812-6 [pii] 10.1016/j.febslet.2009.10.036 (2009).

2. de Sousa Abreu, R., Penalva, L. O., Marcotte, E. M., \& Vogel, C. Global signatures of protein and mRNA expression levels. Mol Biosyst. 5, 1512-1526, doi:10.1039/b908315d (2009).

3. Keene, J. D. RNA regulons: coordination of post-transcriptional events. Nat Rev Genet. 8, 533-543, doi:nrg2111 [pii] 10.1038/nrg2111 (2007).

4. Komili, S., \& Silver, P. A. Coupling and coordination in gene expression processes: a systems biology view. Nat Rev Genet. 9, 38-48, doi:nrg2223 [pii] 10.1038/nrg2223 (2008).

5. Schwanhausser, B. et al. Global quantification of mammalian gene expression control. Nature. 473, 337-342, doi:nature10098 [pii] 10.1038/ nature10098 (2011).

6. Sonenberg, N., \& Hinnebusch, A. G. Regulation of translation initiation in eukaryotes: mechanisms and biological targets. Cell. 136, 731-745, doi:S0092-8674(09)00090-7 [pii] 10.1016/j.cell.2009.01.042 (2009).

7. Rolfe, D. F., \& Brown, G. C. Cellular energy utilization and molecular origin of standard metabolic rate in mammals. Physiol Rev. 77, 731-758 (1997).

8. Johnson, L. F., Levis, R., Abelson, H. T., Green, H., \& Penman, S. Changes in RNA in relation to growth of the fibroblast. IV. Alterations in theproduction and processing of mRNA and rRNA in resting and growing cells. J Cell Biol. 71, 933-938 (1976).

9. Fabian, M. R., Sonenberg, N., \& Filipowicz, W. Regulation of mRNA translation and stability by microRNAs. Annu Rev Biochem. 79, 351-379, doi:10.1146/annurev-biochem-060308-103103 (2010).

10. De Benedetti, A., \& Graff, J. R. elF-4E expression and its role in malignancies and metastases. Oncogene. 23, 3189-3199, doi:10.1038/ sj.onc.1207545 1207545 [pii] (2004).

11. Roux, P. P., \& Topisirovic, I. Regulation of mRNA translation by signaling pathways. Cold Spring Harb Perspect Biol. 4 , doi:cshperspect.a012252 [pii] 10.1101/cshperspect.a012252 (2012). 
12. Ron, D., \& Harding, H. P. Protein-folding homeostasis in the endoplasmic reticulum and nutritional regulation. Cold Spring Harb Perspect Biol. 4, doi:4/12/a013177 [pii] 10.1101/cshperspect.a013177 (2012).

13. Silvera, D., Formenti, S. C., \& Schneider, R. J. Translational control in cancer. Nat Rev Cancer. 10, 254-266, doi:nrc2824 [pii] $10.1038 /$ nrc2824 (2010).

14. Proud, C. G. mTOR Signalling in Health and Disease. Biochem Soc Trans. 39, 431-436, doi:BST0390431 [pii] 10.1042/BST0390431 (2011).

15. Topisirovic, I., \& Sonenberg, N. mRNA Translation and Energy Metabolism in Cancer: The Role of the MAPK and mTORC1 Pathways. Cold Spring Harb Symp Quant Biol. doi:sqb.2011.76.010785 [pii] 10.1101/sqb.2011.76.010785 (2011).

16. Pavitt, G. D., \& Proud, C. G. Protein synthesis and its control in neuronal cells with a focus on vanishing white matter disease. Biochem Soc Trans. 37, 1298-1310, doi:BST0371298 [pii] 10.1042/BST0371298 (2009).

17. Abe, M., \& Bonini, N. M. MicroRNAs and neurodegeneration: role and impact. Trends Cell Biol. 23, 30-36, doi:S0962-8924(12)00165-1 [pii] 10.1016/j.tcb.2012.08.013 (2013).

18. Kasinath, B. S. et al. Regulation of mRNA translation in renal physiology and disease. Am J Physiol Renal Physiol. 297, F1153-1165, doi:90748.2008 [pii] 10.1152/ajprenal.90748.2008 (2009).

19. Zoncu, R., Efeyan, A., \& Sabatini, D. M. mTOR: from growth signal integration to cancer, diabetes and ageing. Nat Rev Mol Cell Biol. 12, 21-35, doi:nrm3025 [pii] 10.1038/nrm3025 (2011).

20. Schneider-Poetsch, T. et al. Inhibition of eukaryotic translation elongation by cycloheximide and lactimidomycin. Nat Chem Biol. 6, 209-217, doi:nchembio.304 [pii] 10.1038/nchembio.304 (2010).

21. Larsson, O., Tian, B., \& Sonenberg, N. Toward a genome-wide landscape of translational control. Cold Spring Harb Perspect Biol. 5 a012302, doi:cshperspect.a012302 [pii] 10.1101/cshperspect.a012302 (2013).

22. Ingolia, N. T., Ghaemmaghami, S., Newman, J. R., \& Weissman, J. S. Genome-wide analysis in vivo of translation with nucleotide resolution using ribosome profiling. Science. 324, 218-223, doi:1168978 [pii] 10.1126/science.1168978 (2009).

23. Larsson, O., Sonenberg, N., \& Nadon, R. Identification of differential translation in genome wide studies. Proc Natl Acad Sci U S A. doi:1006821107 [pii] 10.1073/pnas.1006821107 (2010).

24. Larsson, O. et al. Distinct perturbation of the translatome by the antidiabetic drug metformin. Proc Natl Acad Sci U S A. 109, 8977-8982, doi:1201689109 [pii] 10.1073/pnas.1201689109 (2012).

25. Colman, H. et al. Genome-wide analysis of host mRNA translation during hepatitis C virus infection. J Virol. 87, 6668-6677, doi:JVI.00538-13 [pii] 10.1128/JVI.00538-13 (2013).

26. Larsson, O., Sonenberg, N., \& Nadon, R. anota: Analysis of differential translation in genome-wide studies. Bioinformatics. 27, 1440-1441, doi:btr146 [pii] 10.1093/bioinformatics/btr146 (2011).

27. Gandin, V. et al. Degradation of Newly Synthesized Polypeptides by Ribosome-Associated RACK1/c-Jun N-Terminal Kinase/Eukaryotic Elongation Factor 1A2 Complex. Mol Cell Biol. 33, 2510-2526, doi:MCB.01362-12 [pii] 10.1128/MCB.01362-12 (2013).

28. Warner, J. R., Knopf, P. M., \& Rich, A. A multiple ribosomal structure in protein synthesis. Proc Natl Acad Sci U S A. 49, 122-129 (1963).

29. Gierer, A. Function of aggregated reticulocyte ribosomes in protein synthesis. J Mol Biol. 6, 148-157 (1963).

30. Wright, G. W., \& Simon, R. M. A random variance model for detection of differential gene expression in small microarray experiments. Bioinformatics. 19, 2448-2455 (2003).

31. Eggers, D. K., Welch, W. J., \& Hansen, W. J. Complexes between nascent polypeptides and their molecular chaperones in the cytosol of mammalian cells. Mol Biol Cell. 8, 1559-1573 (1997).

32. Grosso, S. et al. PKCbetall modulates translation independently from mTOR and through RACK1. Biochem J. 415, 77-85, doi:BJ20080463 [pii] 10.1042/BJ20080463 (2008).

33. Oh, W. J. et al. mTORC2 can associate with ribosomes to promote cotranslational phosphorylation and stability of nascent Akt polypeptide. EMBO J. 29, 3939-3951, doi:emboj2010271 [pii] 10.1038/emboj.2010.271 (2010).

34. Zinzalla, V., Stracka, D., Oppliger, W., \& Hall, M. N. Activation of mTORC2 by association with the ribosome. Cell. 144, 757-768, doi:S0092-8674(11)00128-0 [pii] 10.1016/j.cell.2011.02.014 (2011).

35. Bjur, E. et al. Distinct translational control in CD4+ T cell subsets. PLoS Genet. 9, e1003494, doi:10.1371/journal.pgen.1003494 PGENETICS-D-13-00118 [pii] (2013).

36. Leek, J. T., \& Storey, J. D. Capturing heterogeneity in gene expression studies by surrogate variable analysis. PLoS Genet. 3, 1724-1735, doi:07-PLGE-RA-0237 [pii] 10.1371/journal.pgen.0030161 (2007).

37. Guttman, M., Russell, P., Ingolia, N. T., Weissman, J. S., \& Lander, E. S. Ribosome Profiling Provides Evidence that Large Noncoding RNAs Do Not Encode Proteins. Cell. 154, 240-251, doi:S0092-8674(13)00711-3 [pii] 10.1016/j.cell.2013.06.009 (2013).

38. Hsieh, A. C. et al. The translational landscape of mTOR signalling steers cancer initiation and metastasis. Nature. 485, 55-61, doi:nature10912 [pii] 10.1038/nature10912 (2012).

39. Thoreen, C. C. et al. A unifying model for mTORC1-mediated regulation of mRNA translation. Nature. 485, 109-113, doi:nature11083 [pii] 10.1038/nature11083 (2012). 